\title{
ARTICLE
}

\section{Therapeutic communities and planned environments for serious offenders in English prisons}

\author{
Barbara Rawlings \& Rex Haigh
}

\begin{abstract}
Barbara Rawlings has worked in both democratic and addiction therapeutic communities (TCs) on and off since 1972, usually as a researcher. She has conducted a number of qualitative studies of TCs and worked on research and literature reviews of TC history, structures and effectiveness. She is a member of Her Majesty's Prison and Probation Service's Correctiona Services Accreditation and Advice Panel, which assesses psychological programmes for offenders, including TCs, for forensic services in England and Wales. Rex Haigh is a consultant medical psychotherapist in Berkshire, with a particular interest in modified TC treatment programmes for personality disorder. He founded the Community of Communities quality network at the Royal College of Psychiatrists in 2002, and now leads the Enabling Environments Award scheme there. With the 'greencare' team from Slough, which runs a naturebased TC, he won the College's 2014 'Outstanding contribution to sustainability' award
\end{abstract}

Copyright and usage

(C) The Royal College of Psychiatrists 2017.

\section{SUMMARY}

Several English prisons contain democratic therapeutic communities (TCs) for personality disordered offenders, and addiction TCs for serious substance misusers. This article describes how these are organised and comments on how they are specifically tailored and accredited for use in custodial settings. It also describes 'psychologically informed planned environments' (PIPEs), offender pathways for those with personality disorders and psychopathy which provide additional support for psychological treatment. It ends by explaining how 'enabling environments' are assessed, since these are now becoming widely adopted in prisons to reverse toxic environments - which affect staff, the prison and the outside world as well as the individual prisoner - and to counter negative learning found in custodial institutions.

\section{LEARNING OBJECTIVES}

- Understand the key components of treatment in democratic and addiction TCs

- Understand how TCs can operate in a custodial environment

- Appreciate the differences between a planned environment and a psychological treatment programme in a custodial environment

\section{DECLARATION OF INTEREST}

None

To begin with some history, therapeutic communities (TCs) are not new. Their origins can be traced back to the moral treatment of the late 18th century, in particular to the regime established at The Retreat in York. This opened in 1796 and endeavoured to show how treating the insane as normal, as much as this was possible, and giving them useful occupation, could effect improvement in their mental state. In the 20th century, the work of psychiatrists showed how people who had been of sound mind, but were now mentally affected by war, could learn about their condition and even provide therapy for each other. This 'peer treatment' became known during the Second World
War as an effective means for treating 'effort syndrome' (or shell shock). The new therapeutic communities, notably the Cassel in Richmond (Main 1946) and the Henderson in Sutton (Jones 1968), developed from this peer treatment method, and subsequently became places which specialised in the treatment of people with personality disorder. These places gave patients the responsibility for running their own environment, while the staff, instead of directly treating people, developed the skills of enabling people to treat each other. In the late 1950s another type of TC sprang up in California, a self-help programme for drug users (Yablonsky 1965).

This article, though, is about TCs in prisons. HMP Grendon opened as an experimental psychiatric prison for prisoners with antisocial personality disorders in 1962, and is now a TC prison. The Max Glatt Institute ran as a TC at HMP Wormwod Scrubs from 1975 until its closure some 30 years later. Today there is a total of 17 'democratic' TCs in English prisons. Sixteen of these are for men (there are six at Grendon and five at Dovegate) and one is for women. Three of the male TCs are for men who have an intellectual ('learning') disability, which generally means an IQ of less than 70. Overall, the democratic TCs treat around 600 people at any one time, and run according to a single 'core model', of which more later. There are three 'addiction' TCs, all for men, treating 190 residents. To put this into context, the number of convicted adult prisoners with sentences longer than 12 months in March 2017 was nearly 65000 (Ministry of Justice 2017: Table 1.1).

It is useful to emphasise that 'therapeutic community' is a treatment in its own right. Many aspects of the TC, such as large groups, work groups and greater independence, have been adopted by other treatment forms, but this article is about democratic TCs and all the different constituent ingredients that go to make them up. Hopefully the reader will see that their operation is quite difficult to grasp since they seem to accord patients an extraordinary amount of control, which is not 
always welcome in a prison or in any other kind of mental health or addiction establishment, but that is the way they work. For staff, working in a TC in any setting is often a matter of keeping quiet and letting things happen rather than taking over. In a forensic setting, fundamental issues of security and risk mean that staff have to carefully balance this therapeutic requirement to let things happen with the prison requirement to keep control. There can be long, long silences, people can complain they are bored, but the TC way is for patients (and staff) to learn by experience and to learn from their own mistakes and successes. That is not always easy for qualified people who have been trained to take a lead.

\section{Therapeutic community models}

Before turning fully to TCs in prison, some outline needs to be given of TC methods, wherever they are implemented. People tend to work in either:

- democratic TCs (which usually treat people with complex needs, including personality disorder), or

- addiction TCs for those with severe substance misuse problems.

In both, they speak of their model as a 'therapeutic community', as if the other model does not exist. Since democratic TCs fall into the mental health field and addiction TCs specialise in drug addiction, there is not much overlap or moving from one to the other. It is useful therefore to briefly describe the two types, noting that Kennard (1998) offers an excellent and much longer introduction to both.

\section{Democratic TCs}

As has been said, these TCs now specialise in working with people with complex needs, including personality disorder. While this diagnosis is not seen as something which can be cured by treatment, it is seen as something which a person can learn to live with and manage in a healthy rather than an unhealthy way (Dolan 1997). People with personality disorder can be regarded as better therapists for each other than trained mental health workers could be, partly because they know 'from the inside' what it is like to be ruled by a personality disorder, but also because they were nearly always treated in their childhood so abusively by adults, that they cannot trust anyone in authority, and this includes well-meaning therapists.

Democratic TCs are described as having a number of 'pillars' or critical features. Rapoport (1960), who conducted a lengthy ethnographic observation of Henderson Hospital, suggested there were four. These were:
- a flattened hierarchy, with all staff and patients being members of the community

- greater democratisation, with voting by all members on most decisions

- public confrontation of patients' behaviour by patients and staff, and often of staff behaviour by patients

- communalism - which means that everybody works together where possible to keep the community going. Cooking, cleaning, eating, policy-making etc. become shared responsibilities, often carried out in work groups.

Wherever possible patients are expected to take the lead, whether this is running a large group or managing the production of a meal. The work of the staff is to 'hold the boundaries' - to pay attention to the quality of communications between people and to see that relationships are supportive rather than destructive. Haigh (2013) described TCs as having five universal qualities:

- belonging

- safety

- openness

- participation

- empowerment.

These are often now used as good criteria for assessing the therapeutic quality of democratic TCs.

The timetable for democratic TCs is organised around a series of daily or weekly meetings, which include community meetings (attended by everybody), small groups (regular small therapy groups each with a fixed membership of named patients and staff) and activity groups (which can be anything from cleaning to organising an event, from gardening to setting new rules). Importantly there is also social time, or informal time, when nothing is necessarily organised. Patients need to learn how to deal with spare time as much as they need to learn anything else.

\section{Addiction TCs}

These began in the late 1950s in California as a self-help programme for drug and alcohol users. Known as Synanon, the movement was headed by Chuck Dederich, himself a recovering alcoholic, and was based loosely on the 12-step programme of Alcoholics Anonymous and the corporate hierarchies of American business organisations (Yablonsky 1965). The programme quickly lost the alcoholics and kept the drug addicts, and became known for a home-grown therapeutic activity called 'The Game'. This allowed anyone to confront anyone else, and was accompanied by rules which would stop unfair confrontations or bids to 'rescue' people from being criticised. Later, 
people from Synanon moved out and set up similar establishments, and ultimately The Game became 'encounter groups'. These are still a mainstay of current addiction TCs worldwide, and are based on the notion that drug addicts have developed strong psychological defences which must be broken through before they can fully begin recovery. The programme, which is now recognisably similar in many institutions worldwide, is described in great detail in De Leon (2000), with some of the recent developments in programmes and additions to client groups documented in Rawlings \& Yates (2001). According to the model, referrals:

- are initially detoxified, since abstinence is seen as the goal of treatment

- join the TC as juniors (often referred to as an induction phase)

- gradually work their way up through the hierarchy of work and treatment to positions of seniority (Phases 1 and 2)

- go out to a job or to education, but continue to live in the TC (Phase 3)

- ultimately graduate from the programme, live outside and go to work or education

- may finally become a member of staff in the programme, even a TC director.

Not everyone completes treatment though, and for both democratic and addiction TCs there will be some attrition, with people leaving early for one reason or another and often in an unplanned way.

\section{Turning to prisons: democratic TCs}

Outside, in the community, where TCs began, a very different funding landscape has resulted in many TCs either closing down or becoming day TCs for between 1 and 5 days a week. Usually a phone and or internet support group is developed so that in virtual terms these TCs can run all day every day, but it is not the same as a full-time residential TC. Today these can mostly be found in boarding school settings, where the children are living, or in prisons where offenders are already in residence and hotel costs are already part of a budget.

\section{The shape of a prison democratic TC}

Imagine then a democratic $\mathrm{TC}$ in a prison. It will be found in a discrete environment, on a wing, with its own collection of cells, group rooms, association room and staff office. The officers, and in some cases civilian staff as well, will be dressed in uniform. A visitor from another wing might immediately notice colourful walls displaying artwork and positive messages, the use of first names for all staff and offenders and a number of residents sitting around in the office chatting to the staff, for on the other wings surnames are used, walls are uniformly painted, artwork is rarely displayed and prisoners stand at the office door to speak to officers, they do not walk in, sit down and chat.

Each day will be timetabled around community meetings, small groups, creative therapies and work groups, as well as the recognisable prison activities of exercise, association, progress reviews, visits and perhaps work and education. Each day, though, is also governed by prison rules, so that the timetable must fit around the times the residents, like all prisoners, are locked up, during the night and at times during the day. Boundaries are paramount - anyone turning up late to a meeting will be challenged, whether it's the newest recruit or the Governor on a visit. There are senior residents and newer residents, a mix which ensures that the culture of the TC is continued even as people leave and people join. Residents may stay for 18 months, which is considered optimal (Newton 1971), or much longer, depending on how well they seem to be progressing, and on other things such as whether there is a place for them elsewhere. Ideally there will be no one on the wing who is not in therapy, but prison movements and overcrowding being what they are, this is never certain in reality.

'Offence paralleling behaviour' and confronting the truth

Unlike the practice on 'normal' wings, the story of a prisoner's offence is known by all, and told and retold by the offender, patchily at first and then more and more accurately and in more and more detail. Eventually everyone knows what the offender did and what led up to the offence, and this provides one of the key therapeutic tools in treatment - identifying and making explicit their 'offence paralleling behaviour' (Jones 1997). Thus, if a person killed another on the outside after losing his temper, any loss of temper on the TC will be publicly noted, discussed and analysed by everyone in the community, with the intention that eventually that offender will begin to identify such behaviour for himself or herself, and learn to moderate it before it gets out of hand.

As is also the case with addiction TCs, offenders have a problem with 'grassing' on each other, and may regard the requirement to confront one another about bad behaviour as just this. It is so contrary to the code of behaviour they have learned in prison and in the criminal community, that it takes time to gather the courage to publicly name another person's delinquent or negative acts, but it is part of the TC approach that such a thing is supportive rather than disloyal. A person who keeps quiet rather than speaking up is not going to help their peer's progress. 


\section{Both constraint and freedom}

Every day brings its joys and frustrations, a good chat with another member, a lack of interest in a vote, a jam session, or a washing machine that still doesn't work, and every day any or all of these small things may be brought up and discussed. It is difficult for everyone, since this is not just a TC, but a TC where there is a custodial culture and prisoners are expected to be dependent, to rely on the staff and the prison systems, to be locked up and subject to disciplinary rules that massively curtail the freedom someone on the outside would take for granted. In the TC, however, prisoners - or residents as they are usually known - are encouraged to take responsibility for themselves, and to deal with issues rather than leave it to others to sort problems out. It is in this atmosphere of constraint and freedom that the TC in a prison operates, with the balance constantly shifting as different managers come and go, different prison rules are made or abandoned, different residents join the TC, and different issues come to the fore.

\section{Creating a culture of healthy attachment}

The TC culture is developed, over time, through training and custom. Both prison staff and civilian staff, once recruited, need to be trained to carry out TC treatment and to encourage and maintain the TC culture. Importantly, once the TC is up and running, the residents should begin to carry the culture and hand it on to newer residents, thus ensuring that the customs and practices are sustained. It takes a long time to get this right, to get the TC established, which is why the loss of a TC is felt to be devastating (Mackenzie 1997). The work taken to set up a new four-wing TC at the private prison HMP Dovegate has been described in detail by Cullen $\&$ Mackenzie (2011). Their book also underlines the importance of understanding the psychological relationship between the TC and the individual. 'Healthy attachment' (p. 183), something generally considered essential to positive development of the individual, may be quite unknown to many residents, and the TC aims for each individual to develop, little by little, a strong and healthy attachment to the place and to the others there in order to want to change and to be able to change. Part of the training of staff is to emphasise how this process of attachment can be encouraged.

\section{Adapting the TC concept for the prison setting}

Cullen (1997) asked whether it was possible for a prison to be a therapeutic community and, basing his analysis on his experience of working in Grendon, slightly rewrote Rapoport's four pillars of the TC so that they were more suitable for the prison environment. For example, flattening the hierarchy is still possible, but prison officers in the TC have a duty to maintain security and discipline, as well as to deliver therapy. Residents vote on many issues, but are informed when one of these needs to be ratified later by staff. Preparing and eating food together is complicated by health and safety issues, contracted-out services, lock-up times and security rules about what people can and cannot give to residents. However, it is usually possible to achieve all these core features of a TC to an extent. The Community of Communities, an organisation which brings together therapeutic communities under the auspices of the Royal College of Psychiatrists, conducts a biennial audit in conjunction with the Prison Service audit (Paget 2015) and provides a regular means of assessing the therapeutic qualities of these places.

Haigh (2010) has questioned how prison TCs like Grendon can survive in a rapidly changing public sector, while Rawlings (1998a) and Stevens (2013) have both looked at how the culture of the TC can flourish in a prison environment. One answer to this is that it takes a good deal of work by the TC staff. They may take part in prison-wide training and activities, for riot control and hostage-taking, to demonstrate that they are 'still' prison officers, even if they are on the TC. They may educate other prison staff through awareness training and general chat when they meet. Where residents mix with prisoners from the other wings, they too can be ambassadors for the TC. The enthusiasm of TC members for their wing and the suspicion of outsiders in the rest of the prison comes and goes, but over time TCs have become more embedded in English prisons, even though many prison staff and offenders may know little about them, since they are few and far between.

\section{The TC's place in the prison system}

At this point it is worth noting how democratic TC treatment fits into the rehabilitation system of the prison service. The Ministry of Justice aims to reduce reoffending through a number of approaches - such as education, housing and employment - as well as a set of offending behaviour programmes, many of which are organised on cognitivebehavioural therapy (CBT) lines and carried out as sessional courses. Like these programmes, democratic TCs address a convicted criminal's offending behaviour, but are largely directed at serious offenders, many of whom have personality disorders and some of whom score highly as psychopathic. Such people would be unlikely to respond greatly to sessional programmes, and so this residential alternative has been developed. Like other offending behaviour programmes, it 
has been accredited by the Correctional Services Accreditation and Advice Panel (CSAAP), a group of prison experts and academics who inspect the written manuals for all accredited programmes to decide whether or not they are likely to reduce reoffending (Maguire 2010). All programmes are reaccredited every 5 years by CSAAP, and audited to ensure that they continue to operate according to the manuals. Interestingly, owing to the large number of prisoners who cannot access offending behaviour programmes because they are unable to fulfil the education criteria, there are now three TCs for people with intellectual disabilities, which run a longer, slower, more contained and more structured version of the accredited core TC model. These are called TC Plus and can be found at Grendon, Dovegate and Gartree prisons.

\section{Addiction TCs in prisons}

The situation with addiction TCs in the prisons is a little different, and this is due to bureaucratic structures as much as anything. Over the past few years, responsibility for the treatment of substance misuse has been taken over by the National Health Service (NHS). Whereas the Ministry of Justice is concerned with reducing reoffending (and sees lower rates of substance addiction as enabling this), the NHS is more concerned with managing the health of substance misusers. Thus, the NHS has less interest in accrediting programmes which reduce reoffending, although there are still some accredited substance misuse programmes in prisons which are the joint responsibility of the Ministry of Justice and the NHS. While the addiction TCs were accredited by CSAAP some years ago, the accreditation has lapsed, and although they still operate as addiction TCs, their management and assessment is different from the democratic TCs. Moreover, they are run in conjunction with an outside provider, which has experience of drug addiction recovery and can offer further drug treatment in prison or in the community, whereas the democratic TCs are run entirely by the prison service.

\section{Current provision}

There are currently three addiction TCs in English prisons - in Garth, Wymott and Holme House each running a programme that lasts about 18 months. These are all male prisons. Like their counterparts in the community, they take in detoxified addicts, who have serious drug problems and often serious offences. Provided they respond positively to the treatment these individuals will be promoted through a series of phases and jobs so that after a year or so they can be in fairly responsible senior resident positions, running work departments, organising meetings, teaching newer residents in seminars and ensuring that the TC continues to thrive and provide appropriate treatment.

\section{The structure of a prison addiction TC}

Addiction TCs are different from the democratic TCs in a number of ways. To begin with, they are based on the model proposed by De Leon (2000) which we described earlier. Thus, the hierarchy is very clear, often displayed on a notice board, and will include all residents. The residents in charge will have been in treatment longer than others and will be in the final phase of treatment. They are accorded more respect and have more say in how things are run. Junior residents' requests and complaints 'go up through the hierarchy' to teach delayed gratification (i.e. a junior member speaks to his immediate work boss, who speaks to his department head, who speaks to the resident coordinator, who may then sort the issue out or speak to a member of staff, and the reply is then sent back through the same channel). All residents are expected to 'act as if' they are the person they would have been if they had not turned to drug misuse and crime. (This requirement is absolutely not present in the democratic TCs.) Thus, the atmosphere can generally feel upbeat, with 'real' feelings of anger, sadness or despondency surfacing mainly during encounter groups and other therapeutic activities. Morning meetings in particular often end with a group game designed to send people away smiling to start the day. Negative behaviour is liable to a 'pull up', a written slip which gets read out at a meeting. Culprits are given some kind of relevant additional work (for example a resident who regularly talks about drugs may be required to give a seminar on why that is a bad idea or to go and talk to named residents about something positive). Further, often difficult, confrontations take place in encounter groups. As in democratic TCs, drug use is not allowed and drug dealing is absolutely forbidden, but as both of these occur now and again there are penalties when they are discovered, such as exclusion, loss of status or allocation of extra work.

\section{When a problem indicates progress}

It is a feature of both democratic and addiction TCs that when things look as if they are going rather badly, they may actually be going according to plan. For example, the discovery of drugs or drugtaking behaviour may look like a failure on the part of the addiction TCs. However, if one or more residents has reported it, or confessed to it, it is 
seen as evidence that the TC is really very healthy, as it shows that people who would normally keep delinquent behaviour hidden are learning that it is healthier for them and for the TC to make it public. If the drug use has been discovered by staff, it is an opportunity to uncover issues that may have been kept secret in the resident group, and to carry out further therapeutic work. It is often said in the TC that when things seem to be going well, there is probably something not so good going on underneath.

\section{Peer support and social learning}

Like the democratic TCs however, there is an emphasis on personal responsibility, and residents are expected to show 'grown up' behaviour showing visitors around the wing and taking on difficult roles. There is an emphasis on 'peer support', especially by and for other residents at the same level, who comprise a resident's formal peer group. Essentially, residents change through 'social learning', finding that the TC becomes a family, with the director, staff and senior residents being seen as good parents and older siblings, and learning, perhaps for the first time, how to behave well. Staff may themselves be graduates of a TC and in recovery, and they provide crucial role models for residents. Recovering addicts demonstrate just by being there that change is possible. Because they have been addicts in the past, they are ideally placed to see through the lies and evasions of people in treatment, since they have done all that themselves many times. However, owing to prison service rules it is very difficult for a person once convicted of a crime to become a staff member of a prison TC, and so graduate staff are usually exusers from programmes in the community who do not have a criminal record.

\section{Overall}

Both addiction and democratic TCs work with the most difficult of offenders. Most have committed serious crimes. Those who are addicted have had their drug habits for many years. For this reason TCs require reasonably lengthy treatment programmes, and the turnover is consequently low. To maintain quality, it is essential that the right offenders are selected for TCs, and the assessment procedures should ensure that only those with the most deep-seated long-standing problems, who are likely to reoffend unless those problems have been addressed, should be chosen. Since the shorter sessional offending behaviour programmes can put people through treatment much faster than the TCs, they can appear more cost-effective, but they are designed to deal with a different group.

\section{Evaluation research}

Evaluation of TCs can be problematic. Reconviction studies note that any direct comparison with the general prison population is likely to be inaccurate, since the TCs target only the most difficult and dangerous individuals. It is unlikely that the researcher could find a control group for a sufficiently robust randomised control trial (RCT) when it is almost impossible to match their residents (Newton 1971). Since treatment is voluntary, all those who could go into them are probably already there, and waiting lists are deliberately kept short so as not to give untimely hope to future residents. On top of this, slow throughput means low numbers of residents and consequently a lack of statistical power.

TCs are probably the only treatment which will have a real effect on this small proportion of the incarcerated population, and research has shown that the treatment is effective. Evaluations both of psychological changes and of behaviour after treatment have been carried out by a number of researchers (Cullen 1997; Rawlings 1998b). A systematic study and meta-analysis of TC effectiveness which examined such research (Lees 2004) concluded that there was a significant improvement after TC treatment. In terms of prison TCs, Stevens (2013) found that a greater amount of research had been conducted for addiction TCs than for democratic TCs, and consequently the evidence base for these was more robust. However, she notes the difficulties associated with carrying out research on democratic TCs, and points out that, where the research has been done, it has shown improvements for some of the most 'damaged, disturbed and dangerous' people in prison (p. 30).

\section{Planned environments in prisons}

Two more recent innovations in English prisons are also derived from TC practice, but do not require full-scale TCs to be established: 'psychologically informed planned environments' (PIPEs) and 'enabling environments' (EEs). Although they are based in the TC tradition, they do not need to have any of the structures required of TCs, such as community meetings, specific psychotherapies or the immersive experience that is sometimes characterised as 'the process'. PIPEs and EEs are not therapies in themselves, but provide enabling spaces for relationships to be used in a positive way.

\section{The Enabling Environments Standards}

In 2007, the Community of Communities quality network at the Royal College of Psychiatrists derived ten core values-based standards: features which distinguish TCs by being demonstrable and 


\section{BOX 1 The Enabling Environments Standards}

$\begin{array}{ll}1 \text { Belonging } & \text { The nature and quality of relationships are of primary importance } \\ 2 \text { Boundaries } & \begin{array}{l}\text { There are expectations of behaviour and processes to maintain and } \\ \text { review them }\end{array} \\ 3 \text { Communication } & \text { It is recognised that people communicate in different ways } \\ 4 \text { Development } & \text { There are opportunities to be spontaneous and try new things } \\ 5 \text { Involvement } & \text { Everyone shares responsibility for the environment } \\ 6 \text { Safety } & \text { Support is available for everyone } \\ 7 \text { Structure } & \text { Engagement and purposeful activity is actively encouraged } \\ 8 \text { Empowerment } & \text { Power and authority are open to discussion } \\ 9 \text { Leadership } & \text { Leadership takes responsibility for the environment being enabling } \\ 10 \text { Openness } & \text { External relationships are sought and valued }\end{array}$

See College Centre for Quality Improvement (2015: pp. 5-9) for the criteria supporting each of the current standards aims, the main aims of the Pathway and thus of PIPEs are not only to reduce offending, but also to improve psychological health and well-being.

PIPEs are whole environments. In prisons they may be confined to one wing, and run as a small dedicated unit. However, in the community the PIPE extends to entire approved premises, which are not technically units. Consequently, in Her Majesty's Prison and Probation Service (HMPSS) PIPEs are generically referred to as 'services'. Prison and probation staff who work in these services receive training in relational practice, and mental health staff - usually psychologists - are attached as members of clearly defined and wellled teams (Turley 2013; Turner 2015). In addition to staffing and training elements, the programme includes working towards and attaining the Enabling Environments Award (see below). The initiative has an active research programme, from which early findings are encouraging, particularly in terms of fewer violent incidents on prison wings, and improved staff and offender morale (Bond 2014; Turley 2013). In 2017, 23 PIPEs were in operation or were in the process of being set up in prisons and in approved premises.

\section{A supportive environment not a treatment programme}

PIPEs are not in themselves treatment programmes. A PIPE is an element of the OPD Pathway through prison and probation, and is specially designed to contain and support prisoners with complex needs and those likely to have personality disorder. Prisoners who are selected to enter a PIPE unit may leave it to enter a treatment programme such as a TC, and could return to a PIPE when they have finished there. If this is in prison, they would attend education or work sessions from there, like any other prisoner. If it is in a community PIPE, they may well go off to work if they have a job.

People with personality disorder may be particularly thrown off balance by moving from a treatment environment to conventional custody (Livesley 2003), and PIPEs aim to provide a setting in which a person can be helped to build on the learning that takes place in programmes. Thus, they support treatment rather than deliver it. Overall, PIPEs attempt to smooth transitions through an offender's sentence, identify relevant treatment programmes and prepare people for these, enable progression through a sentence and support ex-prisoners after release. In many ways then, PIPEs work with the most difficult and disruptive prisoners from mainstream incarceration and at the same time offer them the kind of support which can help them through 
a pathway of intervention, something which mainstream custodial environments are not always psychologically equipped to provide.

In this way the PIPEs aim to reduce the reoffending rate of those who are probably the most likely to reoffend, because of intractable personality disorders or other complex mental illness or intellectual disability, and at the same time they aim to improve their mental health and well-being. Thus, for example, they aim to reduce serious sexual or violent offending (usually for men), reduce repeated offences (usually for women), enhance relationships and improve institutional behaviour, a change that in itself can lead to better learning on treatment programmes.

\section{Working in a PIPE}

In prison, all staff involved with PIPEs are trained and supervised to promote a psychologically supportive environment, and this means they need to tread a careful path between the theories of psychotherapy, particularly those relating to attachment theory and relationships (Jacobs 1995), and the required discipline and security work of running a wing. PIPEs are explicitly structured to protect their relational environments, since despite the work they do to promote harmonious prison regimes, they are not a conventional criminal justice setting and can present a tension 'both culturally and systematically' (Turner 2015: p. 42). Again, as with the TCs, the real daily work of staff will include managing this tension, and both residents and staff can find themselves ambassadors for a system more likely to be criticised than admired.

Although the PIPEs do not provide treatment, they are organised on structured lines with a number of formal and voluntary groups to enable the development of relationships between all members of the PIPE in different work and leisure circumstances. Individual keyworker sessions are usually provided weekly for each prisoner. On top of this, partnerships may be developed with outside agencies to provide other activities and to open up to the residents the possibilities that they can find positive and nurturing experiences out there in the world.

\section{Enabling environments (EEs)}

Enabling environments in prisons and approved premises, by contrast, are not part of a coordinated development programme, although there is additional support for them within the OPD strategy. Otherwise, Enabling Environments is simply a portfolio-based award that any unit may apply for. Some units may be 'nearly ready' for the award, whereas others need 2 or 3 years of development work to establish and maintain the necessary culture. At the time of writing, 39 prison wings or units have decided to seek to become 'enabling environments' and 83 residential services across the OPD Pathway are engaged in development to meet the Enabling Environments quality standards.

There is an intention by the National Probation Service (part of HMPSS) to develop all approved premises sites in England and Wales to attain the Royal College of Psychiatrists' award. In addition, a small number of entire prisons have been fieldtesting a process to be considered as 'whole prison enabling environments'. This research concludes in 2017. EEs are thus strongly supported by the prison and probation authorities, and an increase in the number of EEs within criminal justice settings in England and Wales can be expected.

\section{Conclusions}

This article has described the TCs that operate for serious offenders and for drug users in English prisons. It has attempted to give some flavour of these and to provide some information about their effectiveness. It has also described the new PIPEs and EEs which are derived from TC core standards and values. PIPEs provide a pathway for those likely to have personality disorder or with the kind of complex psychological problems which makes it especially hard for them to manage in custody or to benefit from treatment opportunities. Both TCs and PIPEs are examples of ways in which the prison service of England and Wales is attempting to work with some of its most difficult prisoners to offer them opportunities to move forward with their lives, and to provide psychologically healthy environments which could make this possible for people who would normally never flourish in a custodial setting. EEs show some promise in helping to reduce negative behaviour and 'toxic cultures' in more mainstream prison settings.

Currently, PIPES and TCs are minority initiatives in the prison system of England and Wales. Like other prison departments, they are isolated from other departments by walls, gates, locks and keys (which do not encourage other staff to visit) and are often seen to be problematic models within a dominant institutional culture concerned with discipline, security and dependence. Nevertheless, they offer intensive and long-term opportunities for prisoners to rethink their criminal way of life and to understand how criminal behaviours took hold. TCs, PIPEs and EEs provide real opportunities for prisoners to benefit from the prison experience and for staff to grow as people within the environments they find themselves providing.
MCO answers

$1 c \quad 2 b \quad 3$ e $\quad 4 d \quad 5 a$ 


\section{References}

Bond N, Gemmell L (2014) Experience of prison officers on a Lifer Psychologically Informed Planned Environment. Therapeutic Communities, 35: 84-94

College Centre for Quality Improvement (2015) Enabling Environments Standards. Royal College of Psychiatrists (http://www.rcpsych.ac.uk/pdf/ EE\%20LS\%20Standards\%20Document\%202015\%202.pdf).

College Centre for Quality Improvement (2017) What is the Enabling Environments Award? Royal College of Psychiatrists (http://www. rcpsych.ac.uk/workinpsychiatry/qualityimprovement/ccqiprojects/ enablingenvironments.aspx). Accessed 16 June 2017.

Cullen E (1997) Can prison be a therapeutic community? The Grendon template. In Therapeutic Communities for Offenders (eds E Cullen, L Jones, R Woodward): 75-99. John Wiley \& Sons.

Cullen E, Mackenzie J (2011) Dovegate: A Therapeutic Community in a Private Prison and Developments in Therapeutic Work with Personality Disordered Offenders. Waterside Press

De Leon G (2000) The Therapeutic Community: Theory, Model and Method. Springer Publishing.

Dolan B, Warren F, Norton K (1997) Changes in borderline symptoms one year after therapeutic community treatment for severe personality disorder. British Journal of Psychiatry, 171: 274-9.

Haigh R (2010) Grendon's contribution to therapeutic communities and personality disorder. Howard Journal of Criminal Justice, 49: 503-12.

Haigh R, Harrison T, Johnson R, et al (2012) Psychologically informed environments and the "Enabling Environments" initiative. Housing, Care and Support, 15: 34-42

Haigh R (2013) The quintessence of a therapeutic environment. International Journal of Therapeutic Communities, 34: 6-15.

Jacobs M (1995) D. W. Winnicott. Sage.

Jones M (1968) Social Psychiatry in Practice. Penguin.

Jones L (1997) Developing models for managing treatment integrity and efficacy in a prison-based TC: the Max Glatt Centre. In Therapeutic Communities for Offenders (eds E Cullen, L Jones, R. Woodward): 12157. John Wiley \& Sons.

Joseph N, Benefield N (2012) A joint offender personality disorder pathway strategy: an outline summary. Criminal Behaviour and Mental Health, 22: 210-7.

Kennard D (1998) An Introduction to Therapeutic Communities. Jessica Kingsley Publishers.
Lees J, Manning N, Rawlings B (2004) Therapeutic community research: an overview and meta-analysis. In A Culture of Enquiry: Research Evidence and the Therapeutic Community (eds J Lees, N Manning, D Menzies, et al): 36-54. Jessica Kingsley Publishers.

Livesley WJ (2003) Practical Management of Personality Disorder. Guilford Press.

Mackenzie J (1997) Glen Parva Therapeutic Community: an obituary. Prison Service Journal, 111: 26

Maguire M, Grubin D, Lösel F, et al (2010) 'What Works' and the Correctional Services Accreditation Panel: taking stock from an inside perspective. Criminology and Criminal Justice, 10: 37-58.

Main T (1946) The hospital as a therapeutic institution. Bulletin of the Menninger Clinic, 10: 66-70.

Ministry of Justice (2017) Offender Management Statistics Bulletin, England and Wales: Quarterly October to December 2016. Ministry of Justice (https://www.gov.uk/government/uploads/system/uploads/ attachment_data/file/610969/offender-management-statistics-bulletinoct-dec-2016.pdf)

Newton M (1971) Reconviction after Treatment at Grendon (Chief Psychologist's Report Series B, No. 1). Home Office, Prison Department. Reprinted (2000) in A Compilation of Grendon Research (ed John Shine): 205-19. Grendon Underwood: HM Prison, Grendon.

Paget S, Thorne J, Das A (eds) (2015) Service Standards for Therapeutic Communities: 9th Edition (CCOl196). Royal College of Psychiatrists' Centre for Quality Improvement

Rapoport R (1960) Community as Doctor. Tavistock Press

Rawlings B (1998a) The therapeutic community in the prison: problems in maintaining therapeutic integrity. Therapeutic Communities, 19: 281-94.

Rawlings B (1998b) Research on Therapeutic Communities in Prisons: A Review of the Literature (http://www.dldocs.stir.ac.uk/documents/ rawlings.pdf).

Rawlings B, Yates R (2001) Therapeutic Communities for the Treatment of Drug Users. Jessica Kingsley Publishers.

Stevens A (2013) Offender Rehabilitation and Therapeutic Communities: Enabling Change the TC way. Routledge.

Turley C, Payne C, Webster C (2013) Enabling Features of Psychologically Informed Planned Environments. National Offender Management Service.

Turner K, Bolger L (2015) The provision of PIPEs - Psychologically Informed Planned Environments. Prison Service Journal, 218: 41-6.

Yablonsky L (1965) The Tunnel Back: Synanon. Macmillan.

\section{MCOs}

Select the single best option for each question stem

1 Therapeutic community treatment:

a is a version of cognitive-behavioural therapy

b should not be continued for more than 3 months

c expects patients to learn responsibility for their behaviour

d can only be provided by qualified professionals

$\mathrm{e}$ is always provided in residential settings.

2 Democratic therapeutic communities:

a have a clear hierarchy for all residents

b can help personality disordered patients to manage their disorders

$c$ are not found in prison settings

$\mathrm{d}$ ensure that patients are kept busy at all times

e minimise upset to patients by discouraging conflict.
3 Addiction therapeutic communities:

a were invented by psychiatrists

b generally require residents to use methadone or a similar substitute drug

c discourage confrontation

d are designed to address problems of mild drug use

e are often staffed by ex-addicts in recovery.

4 Psychologically informed planned environments (PIPEs):

a require a psychiatric report for all participating prisoners

b can originate spontaneously where 'relational practice' is supported

c are treatment programmes

$\mathrm{d}$ involve extra training for prison staff

e are easily established in existing prison regimes.
5 Which of the following statements is not true of enabling environments (EEs)?

a They are specifically for prisoners with a personality disorder diagnosis

b A whole prison can become an enabling environment

c They include therapy groups where prisoners talk about their offences

d They can originate spontaneously where 'relational practice' is supported

e An EE is an award validated by the Royal College of Psychiatrists. 